\title{
Anomalous connection of left atrial appendage with persistent left superior vena cava
}

\author{
L M GERLIS, * J B PARTRIDGE, G I FIDDLER \\ From the Departments of Pathology, Radiology, and Paediatric Cardiology, Killingbeck Hospital, Leeds
}

SUMMARY An unusual connection of the left atrial appendage with a persistent left superior vena cava was demonstrated at angiography in a child with a ventricular septal defect, aortic valve stenosis, and aortic isthmus stenosis; this was later confirmed at necropsy. No previous reports of this anomaly have been found.

The left sided superior vena cava disappears at a late stage both in phylogenetic and embryonic human development and its persistence is a common finding, especially in association with other congenital cardiovascular malformations and it almost invariably drains into the coronary sinus and thence into the right atrium. Occasionally a left sided superior vena cava opens into the left atrium. In the case now reported the anomalous vein entered the coronary sinus and also communicated with the appendage of the left atrium, which was unconnected with the main left atrial cavity.

${ }^{\star}$ LMG is supported by the National Heart Research Fund.

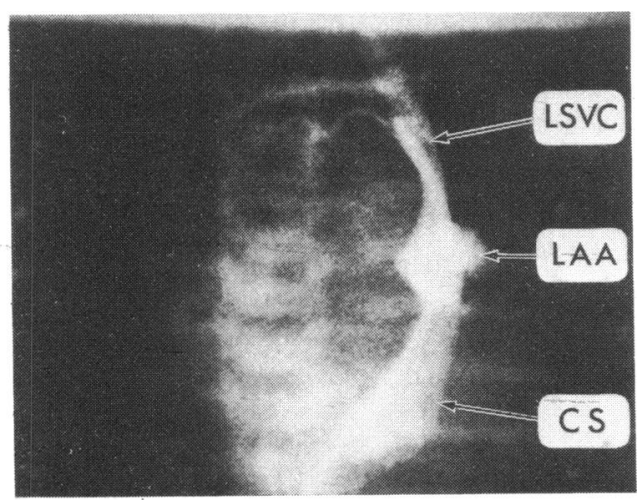

Fig. 1 Left superior vena cava angiogram from a posteroanterior projection. $L S V C$, left superior vena cava; $L A A$, left atrial appendage; $C S$, coronary sinus.

\section{Case report}

This male infant was delivered by caesarean section from a 32-year-old mother suffering from toxaemia of pregnancy, because of fetal distress as shown by tachycardia. Severe hypoglycaemia $(1.2 \mathrm{mmol} / \mathrm{l})$, was present at birth and was treated, and a cardiac murmur was noted. At the age of 1 week there was persistent tachycardia and episodes of central cyanosis were becoming increasingly frequent; the child was then referred to Killingbeck Hospital in cardiac failure.

The clinical, electrocardiographic, radiological, and echocardiographic findings suggested severe aortic valve stenosis. An angiocardiogram on admission showed a left superior vena cava draining into the coronary sinus and into the right atrium. However, and most unusually, just as it crossed the heart border, contrast escaped from it to fill part of a contracting atrium, presumably the left atrial appendage. Aortograms showed isthmus stenosis, between the left subclavian artery origin and the ductus bump, to about half of the normal diameter. The aortic valve was visualised and showed doming consistent with stenosis. At 3 weeks of age an open aortic valvotomy was performed using cardiopulmonary bypass and hypothermia; the heart, however, would not sustain the circulation on repeated attempts to come off bypass.

Necropsy showed no abnormalities apart from those of the cardiovascular system. The heart was of normal size and shape. The right atrium was of normal solitus configuration and received the normal right superior vena cava, the inferior vena cava, and a 


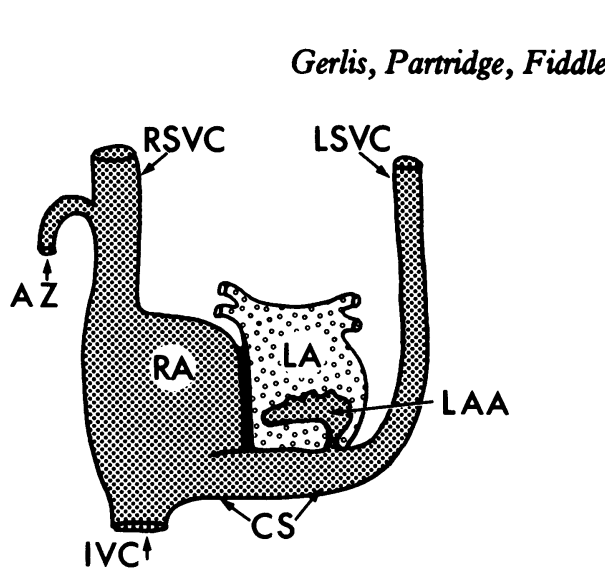

dilated coronary sinus, $3 \mathrm{~mm}$ in diameter. This coronary sinus continued into a persistent left superior vena cava which received the left jugular and subclavian veins; the innominate vein was not shown.

At the junction with the coronary sinus the left superior vena cava communicated through a $1 \mathrm{~mm}$ diameter opening, with a $15 \mathrm{~mm}$ long, $7 \mathrm{~mm}$ wide structure, which had the typical "glove-finger" shape and thin trabeculated wall of a solitus left atrial appendage, but there was no communication between this and the main left atrial cavity. The pulmonary veins were normal and the left atrium was normal apart from the appendicular anomaly. There was slight dysplasia of the medial portion of the tricuspid valve; the atrioventricular valves were otherwise normal.

The ventricles were of normal configuration and equal cavity size and wall thickness; they communicated through a high subcristal septal defect, $3 \mathrm{~mm}$ in diameter, which was immediately below the aortic valve and was partly occluded by the nodular thickened dysplastic portion of the right atrioventricular valve.

The great arteries arose in normal relation from the appropriate ventricles. The pulmonary valve was of normal size, but bicuspid. The aortic valve had been surgically split; it was thickened and nodular and no clear cusp demarcation was evident.

The ascending aorta was $4 \mathrm{~mm}$ in diameter, the arch was to the left, and there was pronounced preductal isthmus stenosis to $1.5 \mathrm{~mm}$ diameter. The aortic branches were normal.

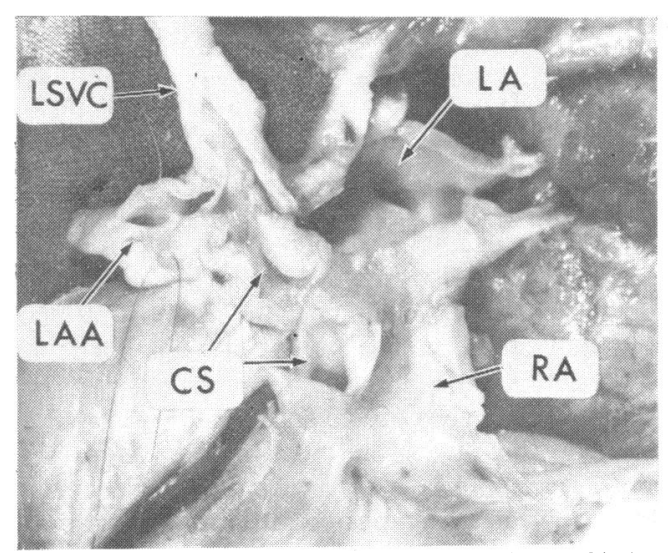

Fig. 2 The upper portion of the heart viewed from behind. LSVC, left superior vena cava; LAA, left atrial appendage (opened); CS, coronary sinus (opened); $L A$, left atrium (opened); $R A$ right atrium.

Fig. 3 Diagram of the atria and great veins. AZ, azygos vein; IVC, inferior vena cava. Other abbreviations as in Fig. 2.

\section{Discussion}

It is difficult to explain this anomaly in terms of embryological maldevelopment. The atrial appendages $\vec{c}$ are derived from the primitive atrial chamber; the absorption of the sinus venosus into the right side of the primitive atrium brings the right atrial appendage into a position adjacent to the opening of the right superior vena cava, which is derived largely from the right lateral extension of the sinus venosus, the Cuvierian duct. The coronary sinus and, when present, the persistent left superior vena cava opening into it, are derived from the left Cuvierian duct and as the mouth of the coronary sinus opens into part of the primitive atrium it is conceivable that there could be circumstances of abnormal growth in which the left atrial appendage could be displaced to that position. In the case here described, however, the left atrial appendage joins the distal part of the coronary sinus at some distance from the right atrial opening and furthermore it has no communication with the otherwise normal left atrium. As a primary malposition appears $\bigcirc$ to be unlikely it is possible that the anomaly represents a discontinuity between the left atrium and its appendage and that the communication between the appendage and the left sided vena caval-coronary sinus junction represents an expanded vein.

We are grateful to Dr O Scott, Dr J B Lynch, and Mr D R Walker for permission to publish details of this case.

Requests for reprints to Dr L M Gerlis, Cardiac Research Unit, Killingbeck Hospital, York Road, $\stackrel{\mathbb{Q}}{\stackrel{\circ}{\circ}}$ Leeds LS14 6UQ. 